\title{
BMJ open Clinical utility of corpus callosum measurements in head sonograms of preterm infants: a cohort study
}

\author{
Agnes Perenyi, ${ }^{1}$ John Amodio, ${ }^{2}$ Joanne S Katz, ${ }^{3}$ Dimitre G Stefanov ${ }^{4}$
}

To cite: Perenyi A, Amodio J, Katz JS, et al. Clinical utility of corpus callosum measurements in head sonograms of preterm infants: a cohort study. BMJ Open 2013;3:e002499. doi:10.1136/bmjopen-2012002499

- Prepublication history for this paper are available online. To view these files please visit the journal online (http://dx.doi.org/10.1136/ bmjopen-2012-002499).

Received 3 January 2013 Revised 24 March 2013 Accepted 26 March 2013

This final article is available for use under the terms of the Creative Commons Attribution Non-Commercial 2.0 Licence; see http://bmjopen.bmj.com

${ }^{1}$ Department of Pediatrics, State University of New York, Downstate Medical Center, Brooklyn, New York, USA ${ }^{2}$ Department of Radiology, State University of New York, Downstate Medical Center, Brooklyn, New York, USA ${ }^{3}$ Physical Therapy Program, State University of New York, Downstate Medical Center, Brooklyn, New York, USA

${ }^{4}$ Scientific Computing Center, State University of New York, Downstate Medical Center, Brooklyn, New York, USA

\section{Correspondence to} Dr Agnes Perenyi; agnes.perenyi@downstate.edu

\section{ABSTRACT}

Objective: To assess the clinical usefulness of measurement of corpus callosum (CC) size in head ultrasound (HUS) to predict short-term neurodevelopmental (ND) outcomes in preterm infants. We hypothesised that including $\mathrm{CC}$ measurements in routine HUS will be an additional tool for early identification of infants at risk of adverse short-term ND outcome, over and above the predictive power of perinatal morbidities.

Design: Retrospective cohort study.

Setting: Level III neonatal intensive care unit (NICU) and outpatient NICU follow-up clinic of an academic medical centre in New York City.

Participants: 929 HUS of 502 infants with gestational age of 23-36 weeks in African-American infants were initially studied. Exclusion criteria included those who died, had gross abnormalities in HUS, infants with race other than African-American, infants with suboptimal quality of HUS, late preterm infants and infants who did not participate in ND follow-up. A total of 173 infants completed the study.

Interventions: CC size (length and thickness) was measured in a subset of 87 infants who had routine HUS between 23 and 29 weeks ( $0-6$ postnatal weeks). Relevant clinical variables were collected from chart reviews. ND assessments were completed in outpatient follow-up clinics. A statistical model was developed to assess the clinical utility and possible predictive value of CC measurements for adverse short-term ND outcome, while adjusting for perinatal morbidities.

\section{Primary and secondary outcome measures:} CC size and ND status.

Results: Measurements of CC size did not add substantial predictive power to predict short-term ND outcome beyond the information provided by the presence of morbidities related to prematurity.

Conclusions: No association was found between morbidities related to prematurity and short-term ND outcome and CC size in preterm infants.

CC measurements in HUS early in life did not have an additional value in predicting short-term ND outcome, therefore did not seem to provide further clinical utility.

\section{INTRODUCTION}

The increasing number and improving survival of preterm infants raises concerns regarding their neurodevelopmental (ND)

\section{ARTICLE SUMMARY}

Article focus

- Preterm infants are at increased risk for complex morbidities and adverse short-term neurodevelopmental (ND) outcome.

- Abnormalities in corpus callosum (CC) size in neuroimaging studies in preterm infants have been linked to impaired neurodevelopment.

- We hypothesised that including CC measurements in routine head sonograms (HUS) studies will be an additional tool for the early identification of infants at risk of adverse short-term ND outcome.

Key message

- Adding CC measurements to HUS studies does not seem to provide further clinical utility to predict short-term ND outcome.

Strengths and limitations of this study

- Predicting ND outcome is important for patients, families and health care providers. It is important to determine the most useful and cost-effective methods for evaluating infants at risk for adverse ND outcome.

outcome. $^{1}{ }^{2} \mathrm{Up}$ to $50 \%$ of the smallest (extremely low birth weight, ELBW $(\leq 1000 \mathrm{~g}$ birth weight)) and very low birth weight infants (VLBW with birth weight of $\leq 1500 \mathrm{~g}$ ) may have major disabilities such as cerebral palsy (CP), sensorineural disabilities and developmental delays with behavioural and learning difficulties at a later age. ${ }^{3}$

Previous studies have shown that in neuroimaging studies, abnormalities in CC size, such as thinning of the CC, have been seen in infants who later developed CP, learning and behavioural difficulties, speech and language delays and cognitive impairment. ${ }^{4-6}$ Anderson $e a^{7} l^{8}$ reported slower CC growth in ELBW infants compared with their intrauterine growth rate in head ultrasound (HUS) studies. Premature infants also seem to have a slower rate of CC growth, ${ }^{9}{ }^{10}$ which ultimately may influence CC size. 
Morbidities associated with prematurity such as retinopathy of prematurity (ROP), bronchopulmonary dysplasia (BPD), patent ductus arteriosus (PDA) and sepsis may also influence brain development, and are therefore often used to predict short-term ND outcome. ${ }^{1-3} 11$ This study aimed to determine if CC size measurements from routine HUS may be clinically useful to predict short-term ND outcome, over and above the predictive power of perinatal morbidities.

\section{PATIENT POPULATION}

Clinical data and HUS imaging studies were analysed in 502 preterm infants (with 929 sonograms, 1-6 sonograms per patient) born at our institution and admitted to the neonatal intensive care unit (NICU) between 2007 and 2009, with gestational age (GA) 23-36 weeks and BW between 545 and $3150 \mathrm{~g}$.

Exclusion criteria included the following patient-related issues: (A) those who died (23 infants with 65 HUS); (B) infants with race other than African-American (10 infants with 23 HUS); and (C) preterm infants $>32$ weeks of GA (125 infants with 139 HUS). Exclusion criteria related to methodology issues included (A) infants with suboptimal quality of HUS (43 infants with 83 HUS) and (B) infants who had gross abnormalities in the HUS, including developmental anomalies of the brain, grades III and IV intraventricular haemorrhage (IVH) and periventricular leukomalacia (17 infants with 57 HUS). This latter group of infants was excluded because the CC could not be clearly visualised and measured, and their pathologies carry their own risk for adverse short-term ND outcome. For the purpose of this study, only those with early HUS (between 23 and 29 weeks of GA or 0-6 postnatal weeks) were analysed, being 87 of the remaining 173 infants. The final study group included 173 infants $<32$ weeks of GA with their 304 HUS. From this cohort, a statistical model was created including a subset of 87 infants, with BW range of 545-1490 g, who had early HUS with CC measurements between 23 and 29 weeks (0-6 postnatal weeks).

\section{METHODS}

The institutional review board at the State University of New York Downstate Medical Center approved this study.

The timing of HUS studies was decided by the neonatologist attending the service. HUS studies were routinely conducted between the first postnatal week of life and term (40+ weeks) age and/or prior to discharge from the NICU as part of the standard of care.

All HUS studies were performed at bedside in the NICU using an ATL 3500 ultrasound machine. The length and thickness of the body of the CC were measured in midline sagittal images by two investigators (AP and JA). The CC length was measured between the extreme margins of the genu and splenium. CC thickness was measured at one-third of this distance, as previously described by Anderson et $a l^{7}$ For intra-rater reliability, both the investigators repeated $\mathrm{CC}$ measurements within a 2-week interval on all HUS. The investigator, AP, performed all CC measurements of 502 infants (929 HUS). For inter-rater reliability, every fourth infant's CC measurements were performed by one of the investigators (JA), which included 247 CC measurements of 125 infants.

Clinical data including infant morbidities, results of HUS studies and ND follow-up were collected from chart reviews. Morbidities related to prematurity included BPD (need for supplemental $\mathrm{O}_{2}>35$ weeks of GA), PDA (treated with medication and/or surgery), sepsis proven by positive culture results and ROP diagnosed by paediatric ophthalmologist. All infants' mothers received antenatal steroid treatment, and 26 infants were from multiple births.

ND follow-up assessments were conducted in our outpatient clinic between 18 and 22 months of corrected age and included standard neurological examination, the Denver II Developmental Screening Test $(\mathrm{DDST})^{12}$ and the Cognitive Adaptive Test/Clinical Linguistic and Auditory Milestone Scale (CAT/CLAMS) ${ }^{13}$ according to our standard of care with classification of neurodevelopmental outcome (normal vs adverse outcome) as published previously. ${ }^{14}$ Standard neurological examination was used to assess muscle tone, reflexes and central nervous system integrity. The DDST was used to assess gross motor developmental milestones, whereas the CAT/CLAMS assessed visual/fine motor problem solving and speech and language development.

\section{Statistical analysis}

Inter-rater and intra-rater agreement for the CC length and thickness were determined by intraclass correlation. We investigated if these measurements provide additional predictive power beyond a set of easily available clinical variables. Logistic regression was used to determine if CC length and thickness were associated with short-term ND outcome when adjusted for four perinatal morbidities-ROP, PDA, BPD and sepsis. In addition, all analyses were also adjusted for GA, postnatal time of HUS and gender.

The area under the curve (AUC) (area under the receiver operating characteristic (ROC) curve), as a measure of predictive performance, was used to assess the clinical usefulness of measuring CC length and thickness, beyond the information provided by GA, gender, postnatal time of the sonogram and the presence of morbidities described above. We used the method of DeLong et $a l^{15}$ to determine whether the improvement of the AUC after including CC measurements was statistically significant. Mann-Whitney and $\chi^{2}$ tests were used to compare the infants for whom the short-term ND outcome was recorded to those with missing outcome on several clinical measurements. All statistical analyses were performed with SAS V.9.2 (SAS Institute, Cary, North Carolina, USA). In all the analyses, $\mathrm{p}<0.05$ was considered significant. 
Table 1 Clinical variables of infants included in the study $(\mathrm{N}=87)^{*}$

\begin{tabular}{ll}
\hline Characteristics & N (\%) \\
\hline Male gender & $42(48)$ \\
Bronchopulmonary dysplasia & $28(32)$ \\
Patent ductus arteriosus & $27(31)$ \\
Sepsis & $42(48)$ \\
Retinopathy of prematurity & $21(24)$ \\
\hline${ }^{*}$ Gestational age $\leq 32$ weeks.
\end{tabular}

\section{RESULTS}

Interobserver agreement was 0.89 and intra-rater reliability was 0.78 for measurements of CC length and thickness.

Table 1 shows the clinical variables of the 87 infants included in the study. The results of Mann-Whitney and $\chi^{2}$ tests showed no statistical difference between infants included and those who were excluded with regard to gender $(p=0.36)$ and the occurrence of main morbidities was examined (BPD, $\mathrm{p}=0.26$; PDA, $\mathrm{p}=0.62$; sepsis, $\mathrm{p}=0.65$; ROP, $\mathrm{p}=1.0)$. Adverse short-term ND outcome was found in 32 cases $(36 \%)$ including CP (3), mild global developmental delay (4), gross motor delay (4), speech and language delay (9) and moderate/severe global developmental delay without CP (12). There was no significant difference $(\mathrm{p}=0.82)$ between CC measurements of those with or without adverse short-term ND outcome.

The ROC curves for the two models (with and without CC measurements) adjusted for GA, gender, postnatal age of the sonogram and the clinical variables (BPD, PDA, ROP and sepsis) are presented in figure 1. AUCs are reported by each curve. No significant difference was found between the AUCs $(p=0.39)$.

\section{DISCUSSION}

Contrary to the findings in the current study, several neuroimaging modalities including HUS and brain MRI studies described correlations with decreased CG size and adverse short-term ND outcome including gross motor impairment, cognitive skills and speech and language development. ${ }^{16-18}$ The size of the CC in various studies have been recorded in $\mathrm{mm}$ (length and thickness), $\mathrm{cm}^{3}$ (volume), $\mathrm{mm}^{2}$ (surface areas), ${ }^{19}{ }^{20}$ and/or ratios, both in HUS and in MRI studies. ${ }^{21} 22$ Differences in measuring and imaging methods may make comparison of the results of various studies more complicated.

Alterations of the CC have been addressed by Thompson et $a l^{21}$ who examined VLBW infants' neuroimaging studies using MRI, including the measurement of CC cross-sectional area at term-equivalent age. This study did not show an association between CC size and ND outcome at 2 years of age.

Cooke et $a l^{22}$ observed no significant correlation between ND outcome and imaging study results in their study, although CC measurements and

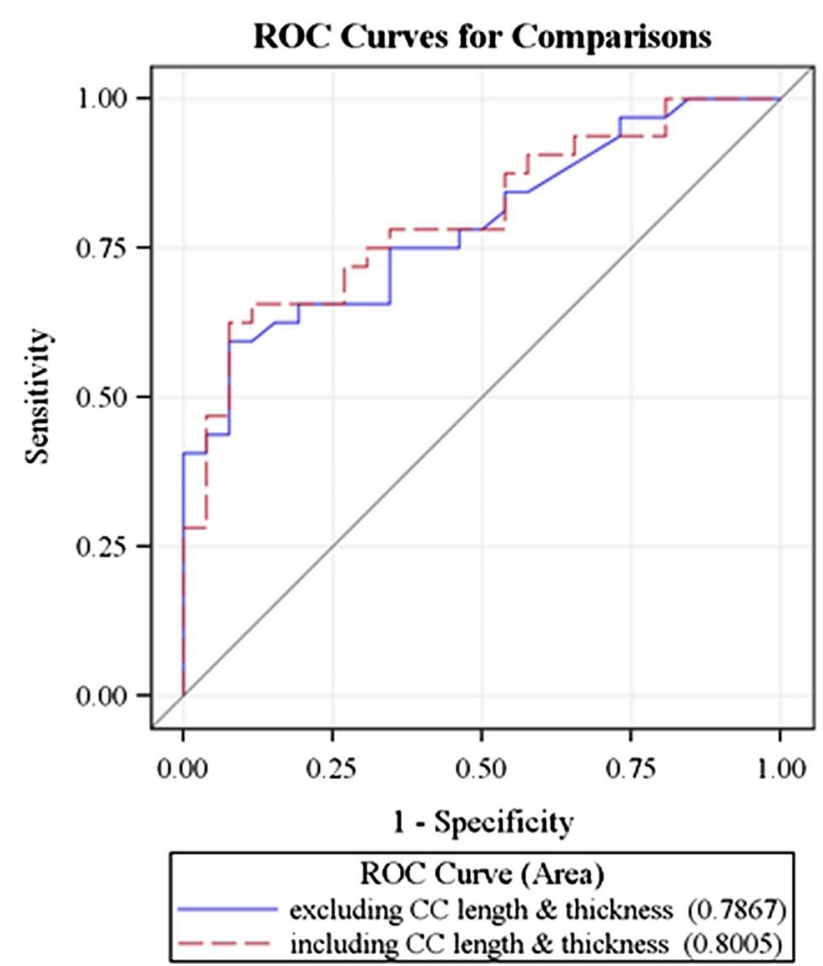

Figure 1 Receiver operator characteristic curves, with and without corpus callosum measurements. Area under the curve $(A \cup C)$ reported for each curve; no significant difference $(p=0.39)$ between the AUCs.

neurodevelopmental assessment have been performed at a different age (15-19 years; long-term outcome) and by different modality (MRI) than what was found in our study.

We chose to assess CC size in HUS studies because of its portability, easy use, reproducibility of measurements and low cost. A further advantage of HUS studies is that infant sedation is not required. We analysed the data of infants between 23 and 29 weeks of GA as a subgroup in a statistical model. These most immature infants frequently have complex morbidities which influence ND outcome.

We had hypothesised that CC measurements would help to predict short-term impaired neurodevelopment based on several studies ${ }^{4-6}$ 9-10 that indicate that abnormalities in CG size are related to adverse short-term ND outcome. However, using the AUC, a widely used measure of predictive performance, analysing ROC curves for comparisons, we found that early HUS studies of CC size (ie, 0-6 weeks postnatally) were not related to short-term adverse outcome.

A limitation of this study is the inclusion of a homogenous (African-American) patient population, so the results may not be applicable for infants of different race or ethnicity.

In summary, inclusion of early CG measurements in routine HUS study evaluations does not seem to have clinical utility with regard to the prediction of adverse short-term ND outcome in preterm infants. CG growth 
rate in HUS may be a better predictor of short-term ND outcome rather than early CC measurements as described by Anderson et l. $^{7} 8$ Diagnostic tools other than neuroimaging studies that may predict ND outcome early in life include Prechtl's general movement assessment, ${ }^{23}$ which is incorporated into the standard of care in our ND follow-up clinic.

Acknowledgements The authors would like to acknowledge the assistance of Jeremy C Weedon, PhD, Associate Director of the Scientific Computing Center at SUNY Downstate, in the statistical analysis of the results.

Contributors AP and JA conceived the idea that corpus callosum measurements in routine head sonograms performed in the neonatal intensive care unit may help to improve the identification of infants at increased risk for poor neurodevelopmental outcome. AP and JA were involved with obtaining corpus callosum measurements. JA read all sonograms as the paediatric radiologist. AP and JSK were involved with neurodevelopmental follow-up of patients. DGS as the biostatistician performed all statistical analyses. All four authors participated in the analysis and interpretation of data and drafting and revision of the article. All authors were involved in the final version of the article to be published.

Funding This research received no specific grant from any funding agency in the public, commercial or not-for-profit sectors.

Competing interests None.

Ethics approval State University of New York Downstate Medical Center Institutional Review Board.

Provenance and peer review Not commissioned; externally peer reviewed.

Data sharing statement No additional data are available.

\section{REFERENCES}

1. Eichenwald EC, Stark AR. Management and outcomes of very preterm low birth weight. N Engl J Med 2008;17:1700-11.

2. Tommiska V, Heinonen $\mathrm{K}$, Lehtonen L, et al. No improvement in outcome of nationwide extremely low birth weight population between 1996-1997 and 1999-2000. Pediatrics 2001;108:134-41.

3. Saigal S, Den Ouden L, Wolke D, et al. School-age outcomes in children who were extremely low birth weight from our international population-based cohorts. Pediatrics 2003;112:943-50.

4. Maneru C, Junque C, Salgado Pineda P, et al. Corpus callosum atrophy in adolescents with antecedents of moderate perinatal asphyxia. Brain Inj 2003;17:1003-9.

5. Nosarti C, Rushe TM, Woodruff PWR, et al. Corpus callosum size and very preterm birth: relationship to neuropsychological outcome. Brain 2004;127:2080-9.
6. Panigrahy A, Barnes PD, Robertson RL, et al. Quantitative analysis of the corpus callosum in children with cerebral palsy and developmental delay: correlation with cerebral matter volume. Pediatr Radiol 2005;35:1199-207.

7. Anderson NG, Laurent I, Woodward LJ, et al. Detection of impaired growth of the corpus callosum in premature infants. Pediatrics 2006;118:951-60.

8. Anderson NG, Laurent I, Cook N, et al. Growth rate of corpus callosum in very premature infants. Am J Neuroradiol 2005;26:2685-90.

9. Caldu X, Narberhaus A, Junque C, et al. Corpus callosum size and neuropsychic impairment in adolescents who were born preterm. $J$ Child Neurol 2006;21:406-10.

10. Narberhaus A, Segarra D, Caldu X, et al. Corpus callosum and prefrontal functions in adolescents with history of preterm birth . J Neuropsychologia 2008;46:111-16.

11. Wilson-Costello $\mathrm{D}$, Friedman $\mathrm{H}$, Minich $\mathrm{N}$, et al. Improved survival rates with increased neurodevelopmental disability for extremely low birth weight infants in the 1990s. Pediatrics 2005;115:997-1004.

12. Frankenburg WK, Dodds J, Archer P, et al. The Denver II-a major revision and restandardization of the Denver Developmental Screening Test. Pediatrics 1992;89:91-7.

13. Kennedy MD, Capute A. The capute scales, CAT/CLAMS instruction manual. Baltimore, MD: Fellows Association, 2006.

14. Perenyi A, Katz JS, Sklar T, et al. Neurodevelopmental outcome and risk factors for impaired development of African American infants in an underserved urban population: a population-based study. J Health Care Poor Underserved 2011;22:983-94.

15. DeLong ER, Delong DM, Clarke-Pearson DL. Comparing the areas under two or more correlated receiver operating characteristic curves: a nonparametric approach. Biometrics 1988;44:837-45.

16. Rademaker KJ, Lam JN, Van Haastert IC, et al. Larger corpus callosum size with better motor performance in prematurely born children. Semin Perinatol 2004;28:279-87.

17. Kulak W, Sobaniec W, Kubas B, et al. Corpus callosum size in children with cerebral palsy: relationship to clinical outcome. J Child Neurol 2007;22:371-4.

18. Narberhaus A, Segarra D, Caldu X, et al. Gestational age at preterm birth in relation to corpus callosum and general cognitive outcome in adolescents. J Child Neurol 2007;22:761-5.

19. Shet RD, Schaefer GB, Keller GM, et al. Size of the corpus callosum in cerebral palsy. J Neuroimaging 1996;6:180-3.

20. lai M, Tanabe J, Goto $M$, et al. A comparative magnetic resonance imaging study of the corpus callosum in neurologically normal children and in children with spastic diplegia. Acta Paediatr 1994;83:1086-90.

21. Thompson DK, Inder TE, Faggian N, et al. Corpus callosum alterations in very preterm infants: perinatal correlates and 2 year neurodevelopmental outcomes. Neuroimage 2012;59:3571-81.

22. Cooke RW, Abernethy LJ. Cranial magnetic resonance imaging and school performance in very low birth weight infants in adolescence. Arch Dis Child Fetal Neonatal Ed 1999;81:F116-21.

23. Einspieler C, Prechtl HFR, Bos AF, et al. Prechtl's method on the qualitative assessment of general movements in preterm, term and young infants. London: Mac Keith Press, 2004. 